\title{
How to Properly Put on and Take off a Disposable Respirator
}

WASH YOUR HANDS THOROUGHLY BEFORE PUTTING ON AND TAKING OFF THE RESPIRATOR.

If you have used a respirator before that fit you, use the same make, model and size.

Inspect the respirator for damage. If your respirator appears damaged, DO NOT USE IT. Replace it with a new one.

Do not allow facial hair, hair, jewelry, glasses, clothing, or anything else to prevent proper placement or come between your face and the respirator.

Follow the instructions that come with your respirator. ${ }^{1}$

\section{Putting On The Respirator}

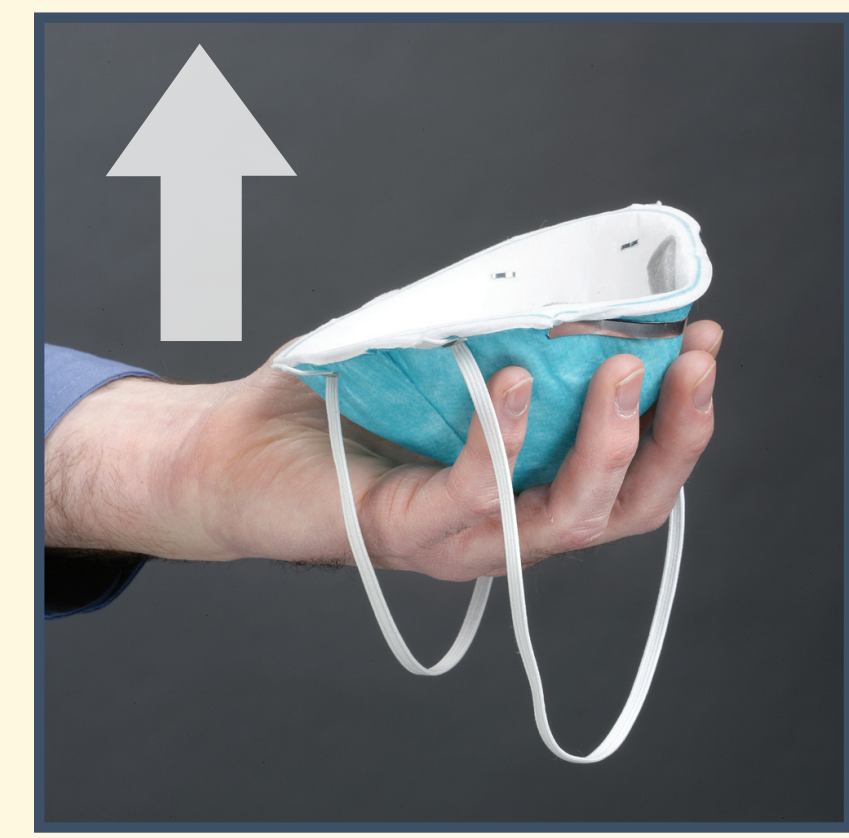

Position the respirator in your hands with the nose piece at your fingertips.

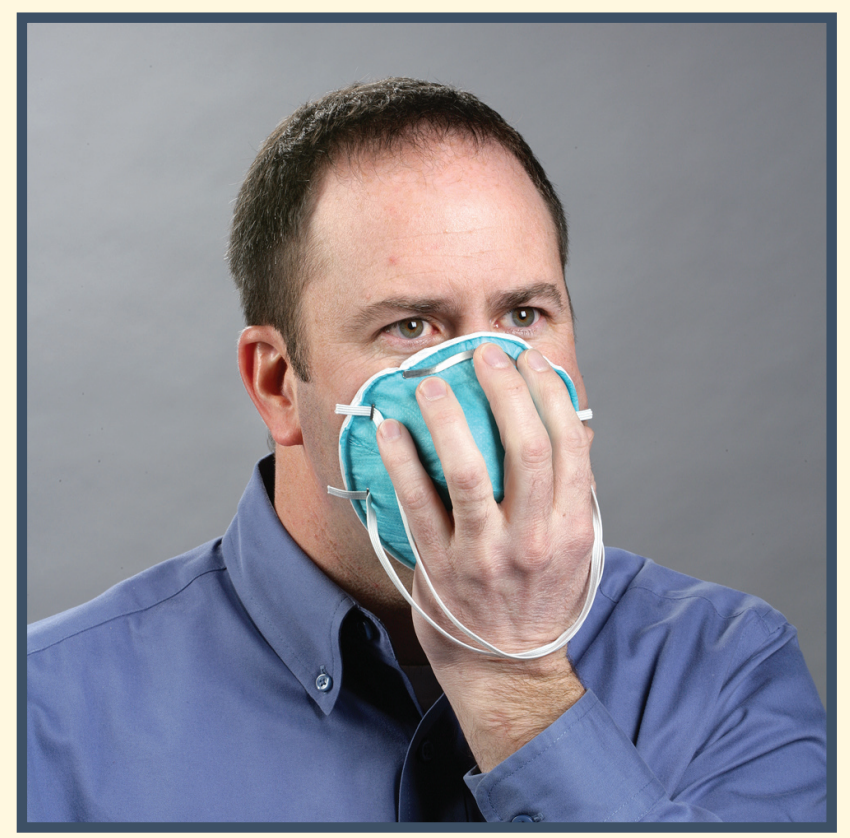

Cup the respirator in your hand allowing the headbands to hang below your hand. Hold the respirator under your chin with the nosepiece up.

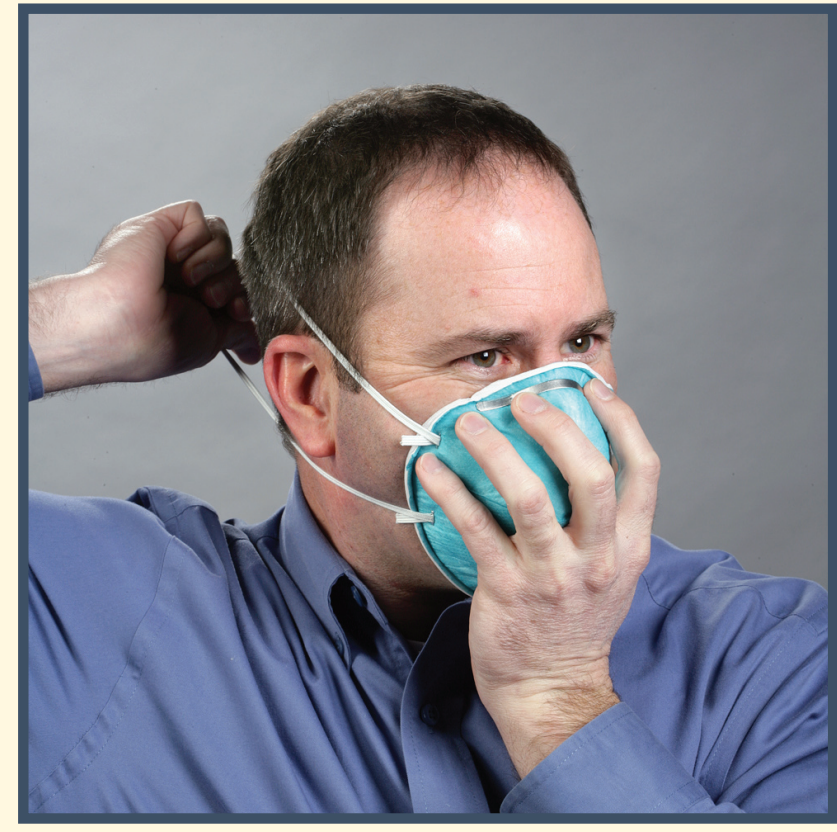

The top strap (on single or double strap respirators) goes over and rests at the top back of your head. The bottom strap is positioned around the neck and below the ears. Do not crisscross straps.

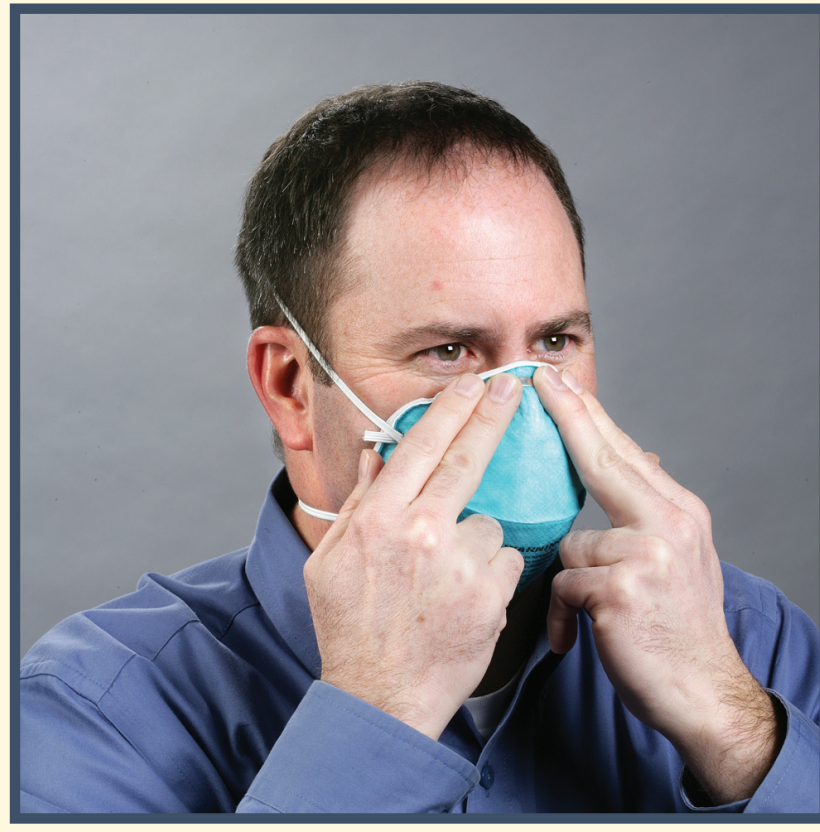

Place your fingertips from both hands at the top of the metal nose clip (if present). Slide fingertips down both sides of the metal strip to mold the nose area to the shape of your nose.

\section{Checking Your Seal ${ }^{2}$}

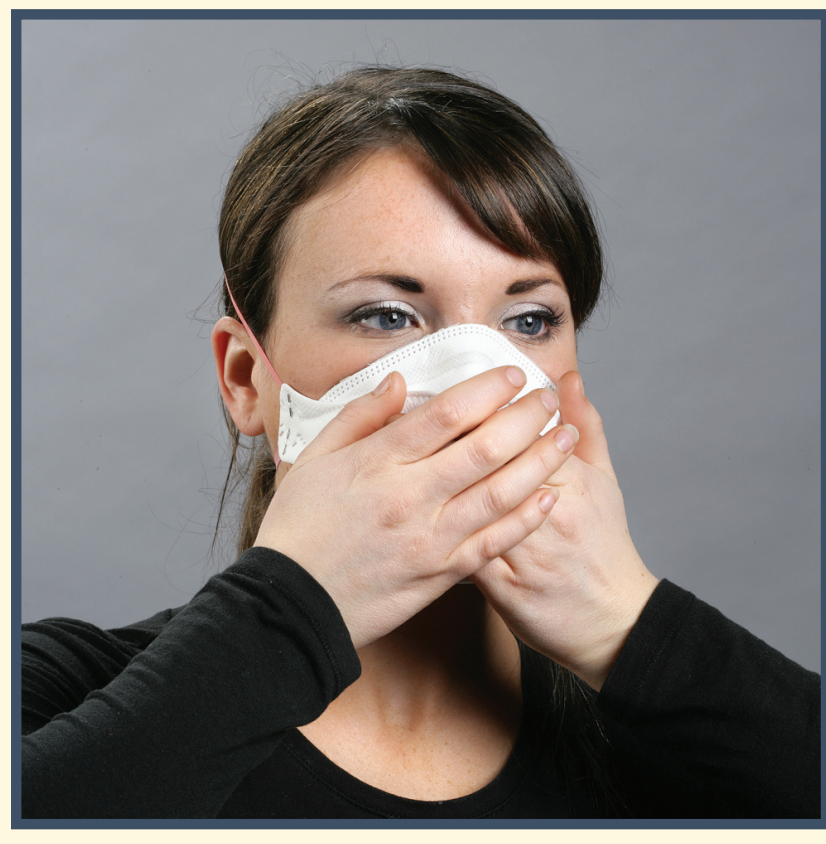

Place both hands over the respirator, take a quick breath in to check whether the respirator seals tightly to the face.

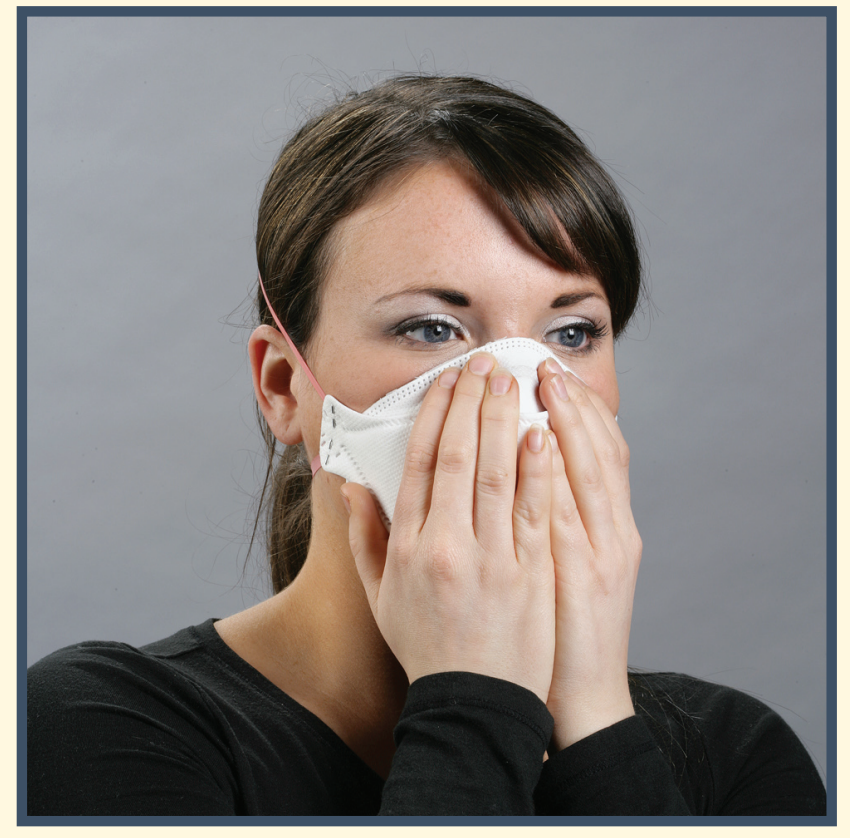

Place both hands completely over the respirator and exhale. If you feel leakage, there is not a proper seal.

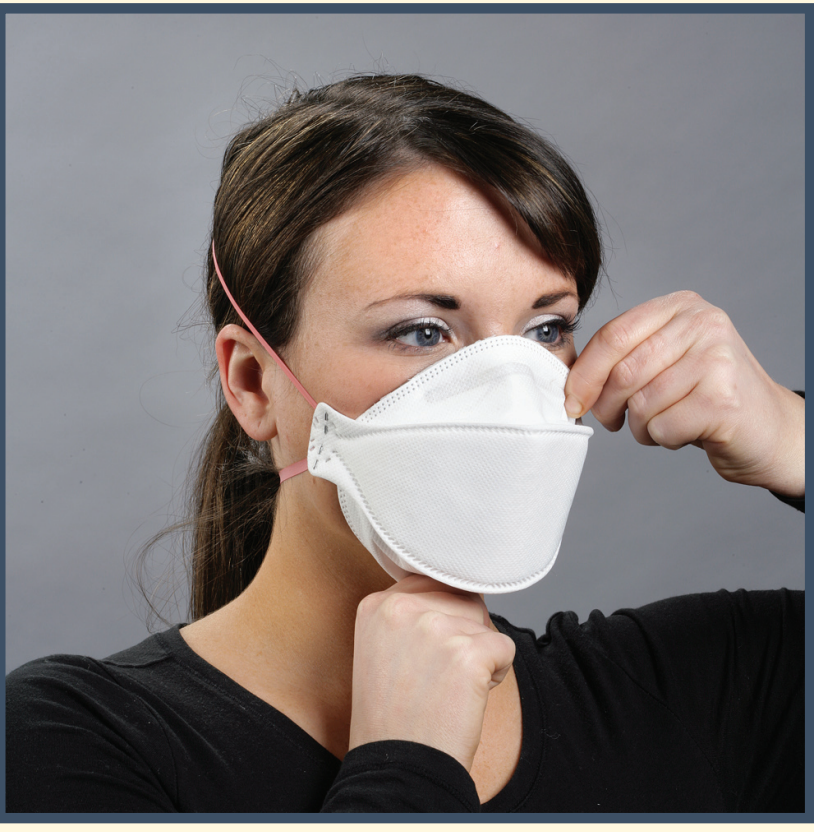

If air leaks around the nose, readjust the nosepiece as described. If air leaks at the mask edges, re-adjust the straps along the sides of your head until a proper seal is achieved.

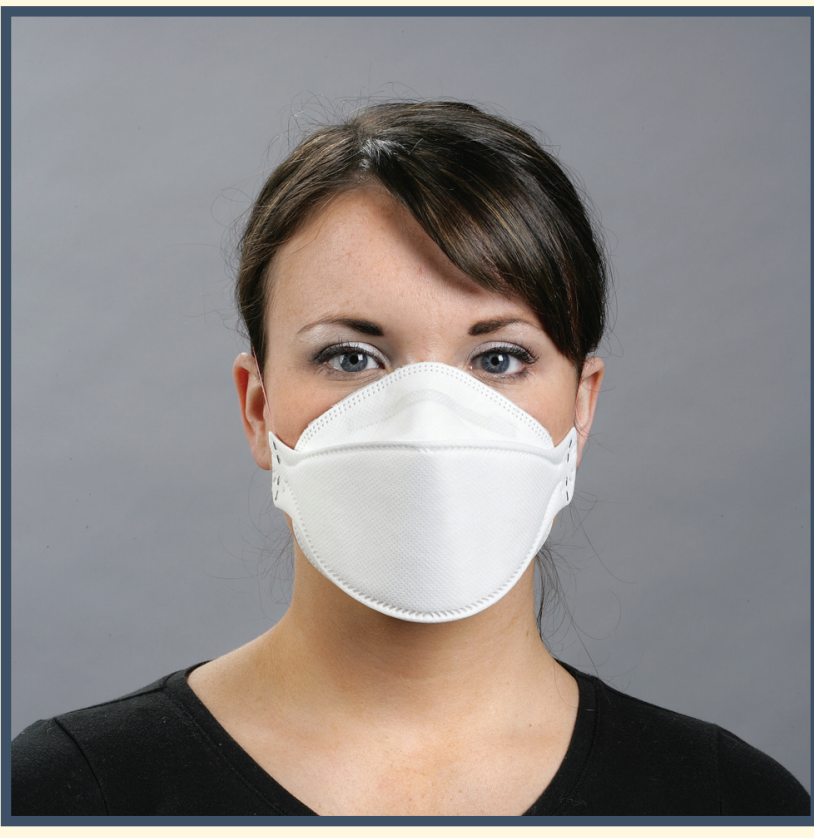

If you cannot achieve a proper seal due to air leakage, ask for help or try a different size or model.

\section{Removing Your Respirator}

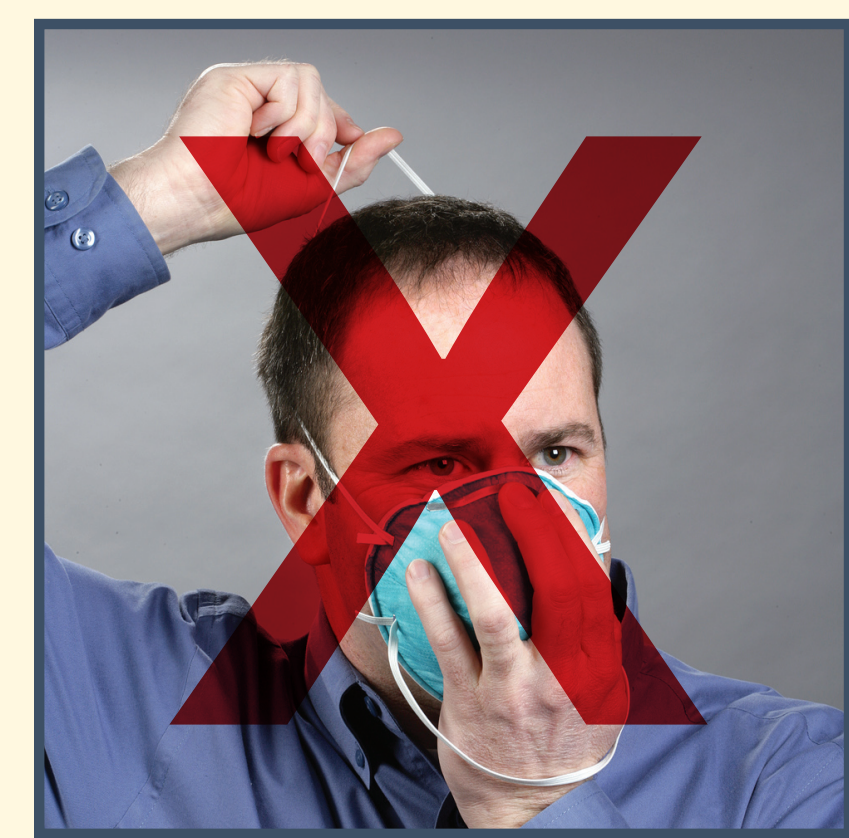

DO NOT TOUCH the front of the respirator! It may be contaminated!

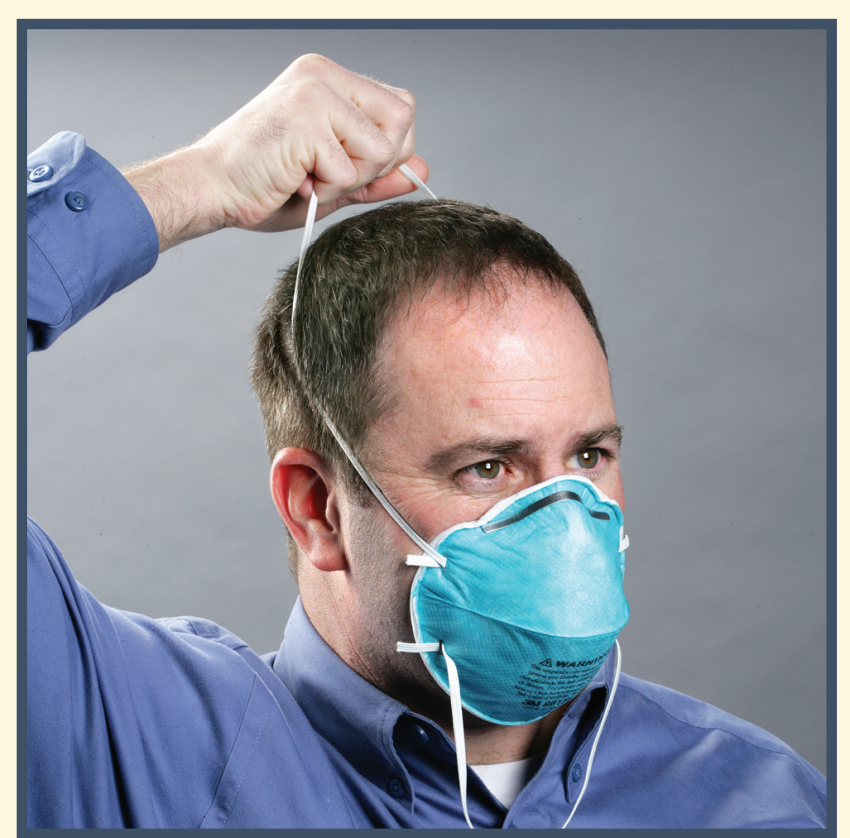

Remove by pulling the bottom strap over back of head, followed by the top strap, without touching the respirator.

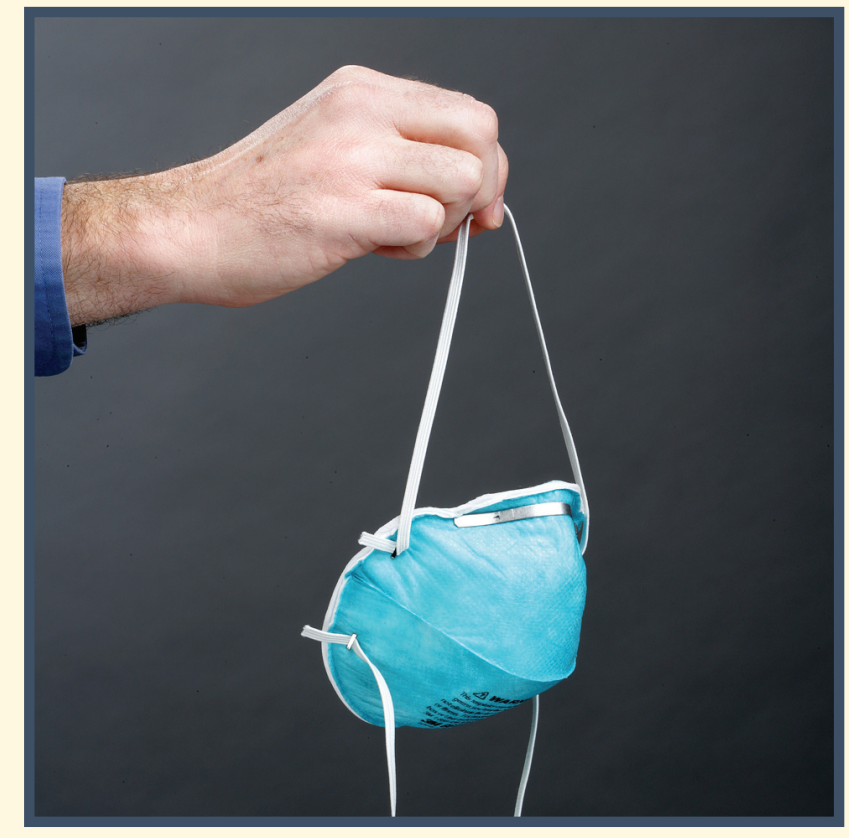

Discard in waste container. WASH YOUR HANDS!

Employers must comply with the OSHA Respiratory Protection Standard, 29 CFR 1910.134 if respirators are used by employees performing work-related duties.

1 Manufacturer instructions for many NIOSH approved disposable respirators can be found

at www.cdc.gov/niosh/npptl/topics/respirators/disp_part/

2 According to the manufacturer's recommendations

For more information call 1-800-CDC-INFO or go to http://www.cdc.gov/niosh/npptl/topics/respirators/

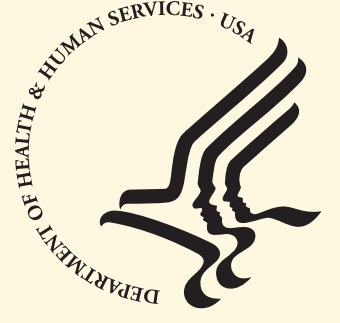

